\title{
Remote Ischemic Preconditioning
}

Limani, Perparim ; Linecker, Michael ; Oberkofler, Christian E ; Barmettler, Gery ; Kaech, Andres ;

Graf, Rolf ; Humar, Bostjan ; Clavien, Pierre-Alain

DOI: https://doi.org/10.1097/SLA.0000000000001765

Posted at the Zurich Open Repository and Archive, University of Zurich ZORA URL: https://doi.org/10.5167/uzh-125982

Journal Article

Published Version

Originally published at:

Limani, Perparim; Linecker, Michael; Oberkofler, Christian E; Barmettler, Gery; Kaech, Andres; Graf, Rolf; Humar, Bostjan; Clavien, Pierre-Alain (2016). Remote Ischemic Preconditioning. Annals of Surgery, 264(5):797-803.

DOI: https://doi.org/10.1097/SLA.0000000000001765 


\title{
Remote Ischemic Preconditioning
}

\section{A Novel Strategy in Rescuing Older Livers from Ischemia-reperfusion Injury in a Rodent Model}

\author{
Perparim Limani, MD, ${ }^{*}$ Michael Linecker, MD, ${ }^{*}$ Christian E. Oberkofler, MD, ${ }^{*}$ Gery Barmettler, $\dagger$ \\ Andres Kaech, PhD, $\dagger$ Rolf Graf, PhD, ${ }^{*}$ Bostjan Humar, PhD, ${ }^{*}$ and Pierre-Alain Clavien, MD, PhD
}

\begin{abstract}
Objectives: The aim of this study was to determine whether remote ischemic preconditioning (RIPC) protects aged liver against ischemia reperfusion (IR). Summary of Background Data: The demands for liver surgery in an aging population are growing. Clamping of vessels to prevent blood loss is integral to liver surgery, but the resulting IR injury (IRI) augments postoperative complications. More so, sensitivity to hepatic IRI increases with age; however, no strategies have been developed that specifically protect old liver. RIPC, a novel protective approach, was performed distant to the surgical site. Whether RIPC may also protect old liver from IRI is unknown.

Methods: RIPC to the femoral vascular bundle was compared against direct ischemic preconditioning (IPC) and the standard of care intermittent clamping (IC) using a model of partial hepatic ischemia in mice aged 20 to 24 months. Liver injury was measured 6 hours after reperfusion. Protective signaling (serotonin-Vegf-Il10/Mmp8 axis, Kupffer cell polarization) was assessed immediately after preconditioning. Neutralizing antibody was used to test the role of Vegf. Hepatic vasculature was examined by electron microscopy. Results: RIPC was superior over other strategies in protecting old liver from IRI, with standard IPC approaches being ineffective. RIPC induced the strongest elevations in circulating Vegf, and Vegf inhibition dampened protective signaling and abrogated the protective effects. RIPC was further associated with improvements in vascular functionality.

Conclusions: RIPC is highly effective in protecting old liver from ischemic insults, mainly owing to its ability to induce circulating Vegf. These findings warrant efforts toward clinical translation.
\end{abstract}

Keywords: intermittent clamping, ischemia reperfusion injury, old liver, remote ischemic preconditioning

From the *Department of Surgery and Transplantation, Swiss Hepato PancreatoBiliary (HPB) and Transplantation Laboratory, University Hospital Zurich, Zurich, Switzerland; and $\dagger$ Center for Microscopy and Image Analysis, University of Zurich, Zurich, Switzerland.

Reprints: Pierre-Alain Clavien, MD, PhD, Department of Surgery and Transplantation, University Hospital Zurich, Raemistrasse 100, CH-8091 Zurich, Switzerland. E-mail: clavien@access.uzh.ch.

Reprints are available through PAC.

This study was supported by the Clinical Research Priority Program "Liver Tumors-from palliation to cure" of the University Hospital Zurich, the Liver and Gastrointestinal Disease Foundation (LGID), and the Swiss National Foundation.

The authors of this manuscript contributed to the study design, analysis, interpretation of data, drafting the article, revising it, and final approval of the version to be published. We confirm that there is no one else who fulfills the criteria but has not been included as an author.

Supplemental digital content is available for this article. Direct URL citations appear in the printed text and are provided in the HTML and PDF versions of this article on the journal's Web site (www.annalsofsurgery.com).

The authors who have taken part in this study declared that they do not have anything to disclose regarding funding or conflict of interest with respect to this manuscript.

Perparim Limani and Michael Linecker shared first authorship.

Bostjan Humar and Pierre-Alain Clavien shared senior authorship.

Copyright (C) 2016 Wolters Kluwer Health, Inc. All rights reserved.

ISSN: 0003-4932/14/26105-0821

DOI: $10.1097 /$ SLA.0000000000001765

\section{(Ann Surg 2016;xx:xxx-xxx)}

schemia and reperfusion injury (IRI) represents a significant source of morbidity and mortality after liver surgery. ${ }^{1}$ Limitation of blood supply - to minimize blood loss during liver parenchyma transection - followed by restoration of perfusion induces an exaggerated inflammatory reaction that eventually leads to neutrophilmediated destruction of liver tissue. ${ }^{2}$ To mitigate ischemic insults and associated postoperative complications, several protective measures have been developed. ${ }^{3,4}$ Although these measures are being used in the clinic, ${ }^{5-8}$ their efficacy in old liver has never been explicitly assessed. This represents a major knowledge gap, because susceptibility to hepatic IRI is known to increase with age. ${ }^{9,10}$ In consideration of an aging population and a growing demand for surgery on elderly liver, ${ }^{11,12}$ a clear need exists to establish measures that protect old liver against IR.

The most common surgical techniques nowadays applied in the clinic to lessen IRI are ischemic preconditioning (IPC) and intermittent clamping (IC) ${ }^{5-8}$ For IPC, liver is exposed to brief episodes of IR via clamping of the portal triad before the actual long ischemia time, as to prepare liver for the imminent hypoxic insult. ${ }^{13}$ IPC clamping either may consist of several repetitive periods or is done for one continuous period. ${ }^{8,14}$ Of note, although continuous IPC has been found to markedly reduce IRI in young mice, it appears to lose this effect in aged liver under both experimental and clinical settings. ${ }^{9,15}$

For IC, the actual ischemic period is interrupted by brief episodes of reperfusion; thus, the protective measure is integral to the surgical operation. IC efficiently protects young mouse liver from IRI and has been shown to outperform IPC particularly in settings of prolonged ischemia. ${ }^{13}$ In the clinic, IC is hence considered the gold standard for ischemic hepatoprotection. ${ }^{16}$ Whether IC can protect old liver remains unclear.

Recently, a novel preconditioning approach has attracted considerable interest. Remote IPC (RIPC) differs from other (pre)conditioning strategies in that it is performed distant from the surgical site, omitting stress on the target organ. Brief episodes of ischemia with intermittent reperfusion are introduced at, for example, a limb, leading to systemic protection against subsequent insults as evinced on kidney, heart, liver, and other tissues. ${ }^{17}$ We have recently identified a general mechanism that underlies the systemic protection conferred through RIPC $^{18}$; remote blood occlusion leads to serotonin release from platelets, which stimulates endothelial Vegf secretion to upregulate in target organs $\mathrm{Il10}$ and Mmp8, acting in concert for protection. Interrupting this serotonin-Vegf-Il10/Mmp8 axis at any point renders RIPC inefficient in protecting against hepatic IR. ${ }^{18}$ Whether RIPC can likewise protect old liver is unclear. However, we have shown that Vegf administration is able to restore the diminished regenerative capacity of old liver to levels seen in young animals. ${ }^{10}$ Therefore, the beneficial effects of RIPC may extend to the protection from hepatic IRI in the elderly. 
Here, we sought to estimate the utility of RIPC as a preconditioning approach for the aging liver patient. First, we compared the hepatoprotective effects of RIPC with IPC (both repetitive and continuous) and the gold standard IC in old mouse liver exposed to IR. Further to this, we assessed whether hepatoprotection through RIPC may rely on its capacity to induce systemic Vegf elevations and associated changes in protective signaling and vascular integrity.

\section{METHODS}

\section{Animals}

C57B1/6 mice (Harlan, Netherlands) aged 20 to 24 months were kept on a 12-hour day/night cycle with free access to food and water. All animal experiments were in accordance with Swiss federal animal regulations and approved by the cantonal veterinary office of Zurich.

\section{Animal Surgery and Treatment}

IPC and IC were performed as previously reported (Fig. 1A). ${ }^{14,19}$ RIPC was done by clamping the femoral vascular bundle just proximal to its confluence with the femoral nerve as described (Fig. 1B). ${ }^{18}$ For IR, an established model of 1-hour $70 \%$ hepatic ischemia was used. ${ }^{18}$ Neutralizing Vegf antibody $(4 \mu \mathrm{g} / \mathrm{kg}$, or unspecific IgG for controls; R\&D Systems, Abingdon UK) was intravenously (i.v.) injected 1 hour before RIPC.

\section{Histological Examination, Immunohistochemistry, and Electron Microscopy}

Hematoxylline-eosine staining was performed on $3 \mu \mathrm{m}$ archived sections. Electron microscopy was performed as reported. ${ }^{10}$ Histological analyses were performed in a blinded fashion.

\section{Biochemical Analysis of Enzyme Activity}

Serum alanine aminotransferase (ALT)/aspartate aminotransferase (AST) levels were measured with a biochemical analyzer (Ektachem DTSCII; Johnson \& Johnson Inc., Rochester, NY).

\section{Transcript Analysis With Quantitative Real-time Polymerase Chain Reaction}

Total RNA was extracted from $50 \mathrm{mg}$ tissue using Trizol reagent (Invitrogen, Basel $\mathrm{CH}$, Switzerland). Quantitative real-time polymerase chain reaction (PCR) was performed on cDNA (ThermoScript reverse-transcription PCR System; Invitrogen) using an ABI Prism 7500 Sequence Detector System (PE Applied Biosystems, Rotkreuz CH, Switzerland). Primers are listed in Suppl. Table 1, http://links.lww.com/SLA/B17. The results represent mean fold induction of mRNA expression $\pm \mathrm{SD}$.

\section{ELISA}

Serotonin (BA E-5900; Rocky Mountain Diagnostics Inc, Colorado Springs, CO), Hmgb1 (ST51011; IBL International, Hamburg, Germany), and Vegf (DY493; R\&D Systems, Minneapolis, $\mathrm{MN}$ ) levels were measured in triplicates by enzyme-linked immunosorbent assay (ELISA).

\section{Statistical Analysis}

GraphPad Prism (GraphPad Software, Inc., La Jolla, CA) was used. Groups were compared with a $t$ test or 1-way analysis of variance (ANOVA) with posthoc Bonferroni correction where appropriate. $P<0.05$ was considered significant. Mean $\pm \mathrm{SD}$ is shown. Six animals/group were analyzed unless otherwise stated.

\section{RESULTS}

The experimental set up is summarized in Fig. 1A and compares RIPC with IC, IPC, and sham proper controls for both

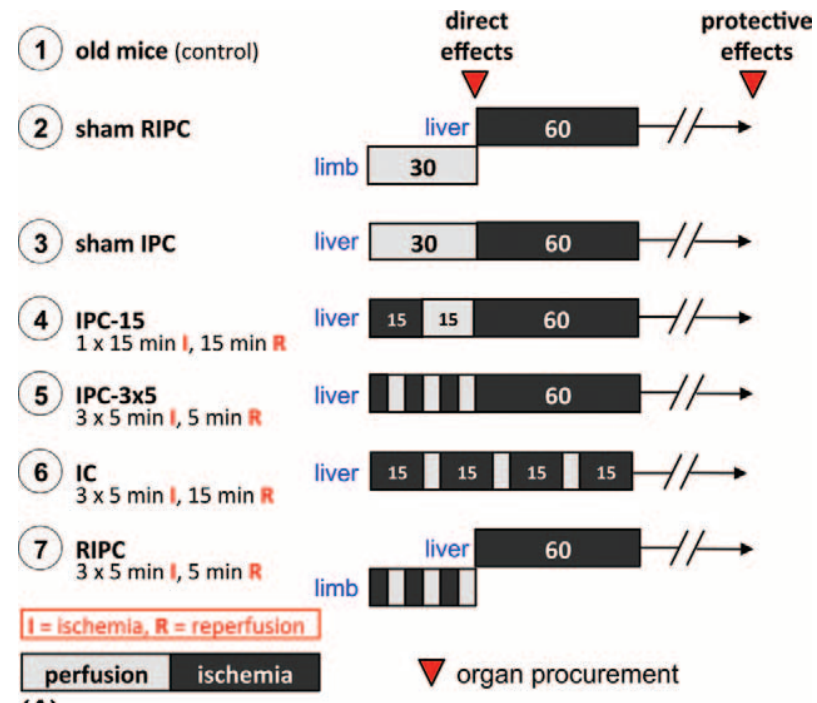

(A)
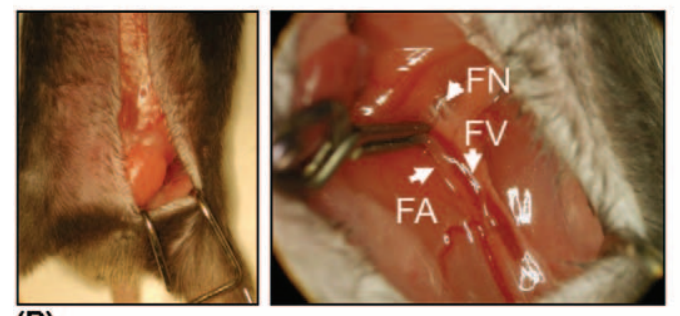

(B)

FIGURE 1. Experimental set up for comparing RIPC with other protective measures. A, Design of experimental procedure. IPC-15 continuous IPC, IPC- $3 \times 5$ repetitive IPC. Save for IC, these procedures comprised the same total exposure to ischemic and reperfusion periods. IC comprised a shorter total ischemic exposure (60 vs $75 \mathrm{~min}$ ) due to its intrinsic design. Sham operations included all steps of either RIPC or IPC apart from vessel clamping. Control animals were not manipulated and served to retrieve baseline values for injury markers. Tissue/ serum was analyzed either $6 \mathrm{~h}$ after reperfusion to assess IRI, or immediately after preconditioning to assess direct effects. For all experimental arms, $\geq 6$ mice/group were used. B, Intraoperative situs for RIPC. FA indicates femoral artery; FN, femoral nerve; FV, femoral vein.

RIPC and IPC, followed by 1 hour of $70 \%$ hepatic ischemia. Each procedure was performed in mice aged 20 to 24 months. Survival for all experimental arms was $100 \%$, enabling the analysis of all animals.

\section{RIPC is Superior in Protecting Old Liver From Ischemic Insults}

To determine which of the preconditioning approaches best protects aged liver against IRI, we measured several injury-related parameters at 6 hours following reperfusion. Compared with sham procedures, IPC was ineffective in reducing the hepatic injury markers AST and ALT (Fig. 2A), necrotic liver area (Fig. 2B), and serum Hmgb1 levels, a generic marker of necrotic cell death (Fig. 2C). IPC with repetitive ischemia performed slightly better than continuous IPC, but remained without significant effects (Fig. 2AC). In contrast, IC lowered all injury parameters following reperfusion, particularly Hmgb1 (Fig. 2A-C). RIPC, on the contrary, 

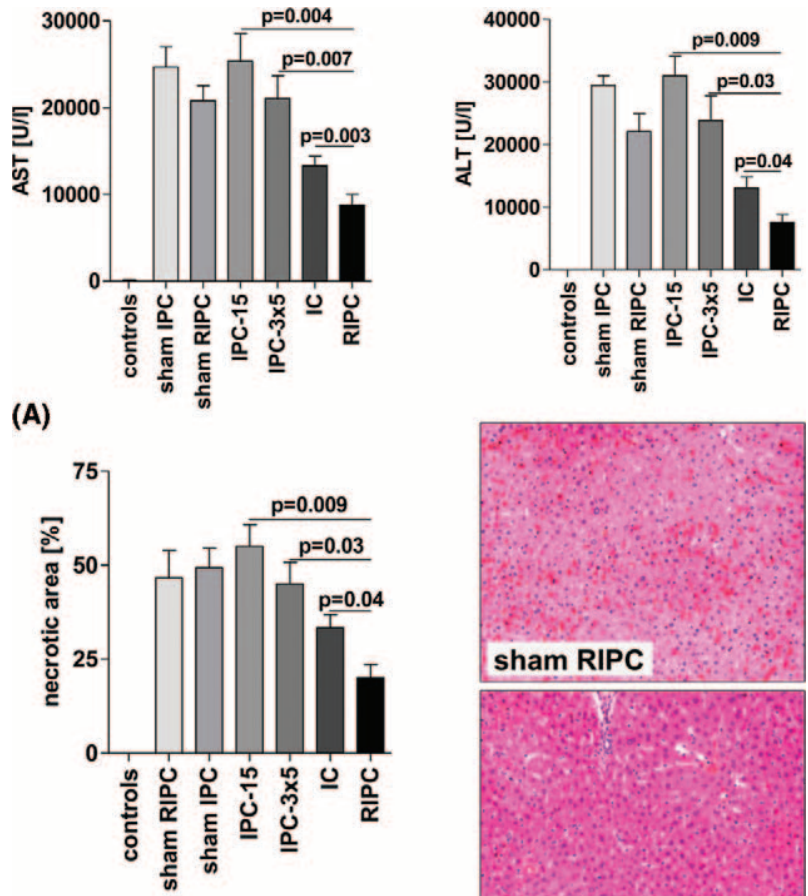

(B)

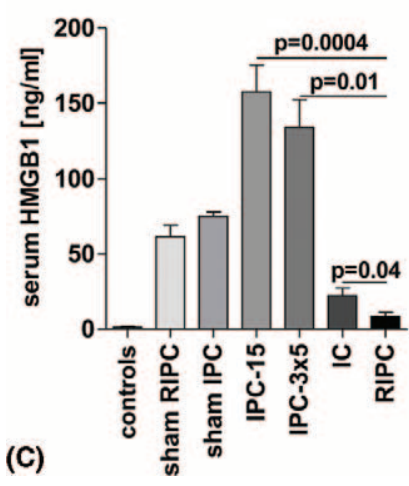

FIGURE 2. IRI levels at $6 \mathrm{~h}$ postreperfusion following (pre)conditioning. A, Serum AST, ALT. B, Necrotic area. C, Serum Hmgb1.

outperformed all other procedures, compared with these, including IC. RIPC significantly reduced serum AST/ALT, necrotic area on histology, and serum Hmgb1 (Fig. 2A-C). Therefore, among the tested procedures, RIPC is the most effective in protecting aged liver from IRI.

\section{RIPC is Superior in Promoting Protective Signaling}

We have previously shown that the RIPC-conferred protection relies on the activation of the serotonin-Vegf-Il10/Mmp8 axis. ${ }^{18}$ To determine whether this axis may contribute to the beneficial effects of RIPC in old liver, we assessed the levels of these molecules immediately after performing preconditioning, that is, before reperfusion. Likewise, we assessed serum Hmgb1, because lowering prereperfusion Hmgb1 levels is strongly protective in IRI. ${ }^{20}$ Furthermore, we measured markers of Kupffer cell polarization, as an M1 $>$ M2 polarization shift can mitigate IRI. ${ }^{21}$ No measurements were done for IC, as this technique does not include a defined prereperfusion period.
Compared with IPC, RIPC induced the highest elevations in serum serotonin and Vegf while markedly reducing Hmgb1 levels (Fig. 3A-C). Indeed, the IPC approaches were statistically indistinguishable from sham controls for serotonin and Vegf, but associated with clear elevations in Hmgb1 (Fig. 3A-C), known to be a key mediator of ischemic injury following reperfusion. ${ }^{20}$ Moreover, although all measures led to Mmp8 induction, Il10 was most potently induced by RIPC (Fig. 3D, to the right). When ranked $I l 10$ and $M m p 8$ expression data were combined into 1 value, RIPC induced the highest Illo/Mmp 8 score (mean \pm SD: $16.96 \pm 2.1$ for RIPC, $8.86 \pm 2.26$ for IPC- $3 \times 15,8.42 \pm 2.31$ for IPC- $15, P<0.001$ ). Therefore, only RIPC will lead to full Il10/Mmp8-mediated protection, as neither Il10 nor Mmp8 alone is sufficient to lessen IRI. ${ }^{18}$ Finally, RIPC markedly reduced the gene expression of M1 polarization markers Emrl, Cd40, Nos2a, and Ill2a (associated with proinflammatory Kupffer cell activity), whereas the IPC approaches had a little effect. Some improvement, particularly with intermittent IPC, was noted for the M2 markers Arg, CleclOa, and Mrcl; however, this was modest compared with RIPC (Fig. 3D). We conclude that RIPC is superior over IPC in inducing the serotonin-Vegf-I110/Mmp8 axis, in reducing Hmgb1 levels, and in promoting M2 Kupffer cell polarization, all molecular events that are associated with protection from IRI.

\section{Vegf is a Key Mediator of Protective RIPC Effects in Old Liver}

In young animals, Vegf is central for the conversion of RIPC stimuli into systemic protection. ${ }^{18}$ To define the role of Vegf in the RIPC effects on old liver, we pretreated aged mice with neutralizing $\alpha$ Vegf antibody before RIPC, leading to pre-reperfusion Vegf levels akin to sham controls (Fig. 4A). Antibody pretreatment further led to an increase in Hmgb1 (Fig. 4A) to levels observed after IPC (Fig. 3A). Moreover, $\alpha$ Vegf treatment prevented the RIPC-induced downregulation of M1-associated genes. Vice versa, M2 markers were dampened to levels seen after IPC, as were the elevations in $I l 10$ expression (Figs. 3B and 4A). Mmp8, which was similarly induced by all preconditioning approaches (Fig. 3B), was not affected by $\alpha$ Vegf pretreatment (Fig. 4A). Nonetheless, $\alpha$ Vegf significantly $(P=0.02)$ reduced combined Illo/Mmp 8 scores relative to RIPC alone (mean \pm SD: $12.6 \pm 2.07$ for RIPC and $\alpha$ Vegf, $17.06 \pm 2.51$ for RIPC).

To define whether $\alpha$ Vegf-induced changes in pre-reperfusion signaling translate into an abrogation of RIPC-induced protection, we treated mice with $\alpha$ Vegf antibody, performed RIPC followed by 1 hour ischemia, and assessed injury 6 hours after reperfusion. Upon $\alpha$ Vegf treatment, RIPC lost all its protective effects, resulting in IRI as seen in sham controls (Fig. 4B). We conclude that Vegf is a central mediator of the protective effects RIPC has on old liver.

\section{RIPC Mitigates Age-related Deficiencies in Hepatic Sinusoids}

Liver sinusoids feature highly differentiated endothelium typified through fenestration. Sinusoidal fenestration is known to disappear with age and negatively impacts liver function. ${ }^{22} \mathrm{We}$ performed RIPC on old mice and prepared liver immediately after the last RIPC cycle for electron microscopy. Although liver of shamtreated mice had typical signs of pseudocapillarization, fenestration in sinusoids of RIPC-treated mice was enlarged and more regular (Fig. 5). Therefore, RIPC mitigates the age-related dedifferentiation of hepatic sinusoids.

\section{DISCUSSION}

In this study, we demonstrate that RIPC is an effective strategy to protect old mouse liver from ischemic injury. Indeed, RIPC 

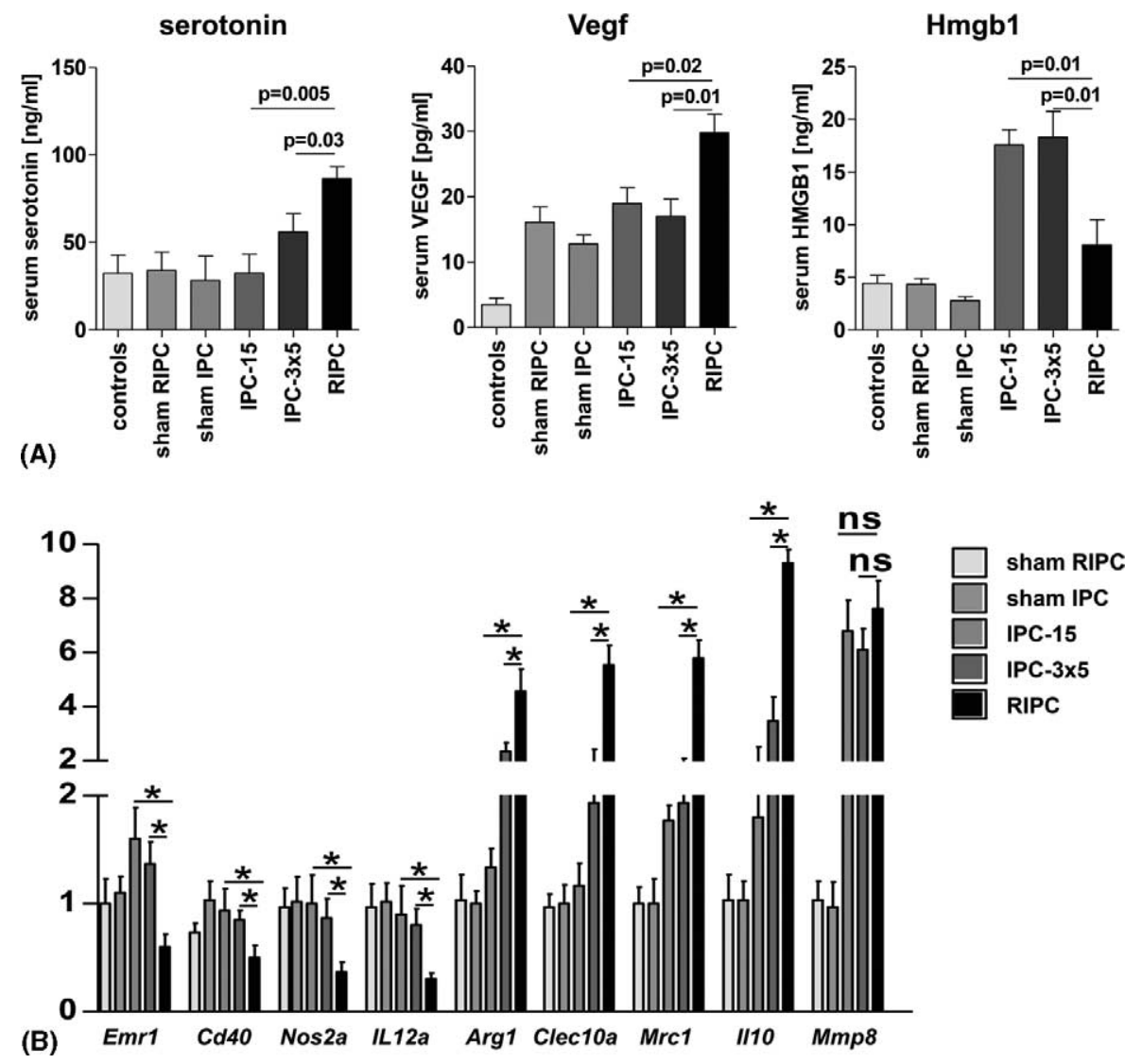

FIGURE 3. Direct effects immediately following (pre)conditioning. A, Effects on circulating serotonin, Vegf, and Hmgb1. B, effects on Kupffer celll polarization markers (M1: Emr1, Cd40, Nos2a, II12a: M2: Arg1, Clec10a, II10) and cytoprotective molecules (II10, Mmp8). ${ }^{*} P<$ 0.05. ns indicates nonsignificant.

performs even better than IC, the current gold standard in the clinic. The superior effects of RIPC are dependent on its ability to induce systemic elevations in Vegf, which in turn activates hepato-protective signaling in liver.

To define the protective capacities of RIPC, we compared with several surgical approaches that are being used in patients to reduce postoperative complications resulting from IR exposure. Liver injury was assessed by multiple parameters at 6 hours postreperfusion, a time point that we have previously found to reliably reflect injury levels present also later after reperfusion. ${ }^{18}$ On all measures, RIPC outperformed IC, while the IPC approaches failed to confer protection on aged liver and were even counterproductive on measures such as Hmgb1, consistent with a previous study. ${ }^{9}$

RIPC alone induced the highest rise in the serotonin-Vegf axis compared with the other approaches. Neutralizing Vegf antibody abolished the RIPC effects, indicating a key role for Vegf in the protection of old liver, and consistent with Vegf as a mediator of systemic RIPC effects. ${ }^{18}$ Vegf antibody also markedly diminished the RIPC-induced changes in M1/M2 polarization markers, suggesting that anti-inflammatory M2 polarization of Kupffer cells contributes to the protection against IR. These effects are akin to those of $\Omega 3$ fatty acids, wherein protection from hepatic IRI is associated with a shift to M2 polarization. ${ }^{21}$ Likewise, Vegf inhibition suppressed the RIPC-induced elevations in Il10, but not those in Mmp8, two important components of the protective serotonin-Vegf axis. ${ }^{18}$ In keeping, Mmp8, but not I110, was induced by the ineffective IPC approaches, confirming that upregulation of both molecules is needed for protection. ${ }^{18}$ On the basis of these data, we propose that circulating Vegf primes old liver against harm likely by shifting Kupffer cells toward an anti-inflammatory repair phenotype. In addition, Vegf promotes the expression of cytoprotective Il10, which - despite being a typical M2 macrophage molecule - is also strongly expressed in hepatocytes after RIPC, wherein its acts in concert with Mmp8 to protect the parenchyme. ${ }^{18}$

RIPC further improved the age-related decline in sinusoidal fenestration. Interestingly, Vegf signaling is required to maintain full fenestration in rat sinusoids, and we have previously shown that serotonin-Vegf signaling improves fenestration in old liver. ${ }^{10,23}$ Of note, fenestration reopening correlated with an improvement of the diminished regenerative capacity of old liver. ${ }^{10}$ Given that recovery from ischemic insults requires parenchymal regeneration, RIPC might hence reduce ischemic injury by promoting hepatocyte regeneration via a mechanism dependent on Vegf, consistent with the key regenerative role of Vegf after hepatectomy. ${ }^{24}$ Further work is required to demonstrate an effect of RIPC on liver regeneration; however, preliminary data from our laboratory suggest that RIPC does promote regeneration after resection.

RIPC likewise elicits a number of other, Vegf-independent, mechanisms that contribute to its beneficial effects, including processes beyond purely ischemic insults. ${ }^{25}$ Although the latter effects are systemic and may affect virtually any organ, ${ }^{17,18,26}$ other benefits of RIPC may extend to processes such as fracture healing, fibrotic prevention, acute mountain sickness, exercise performance, or pancreatitis. ${ }^{18,27-30}$ On the contrary, the variety of potential action mechanisms may pose limits to the clinical application of RIPC. Commonly used anesthetics/analgesics (eg. propofol, fluranes, 

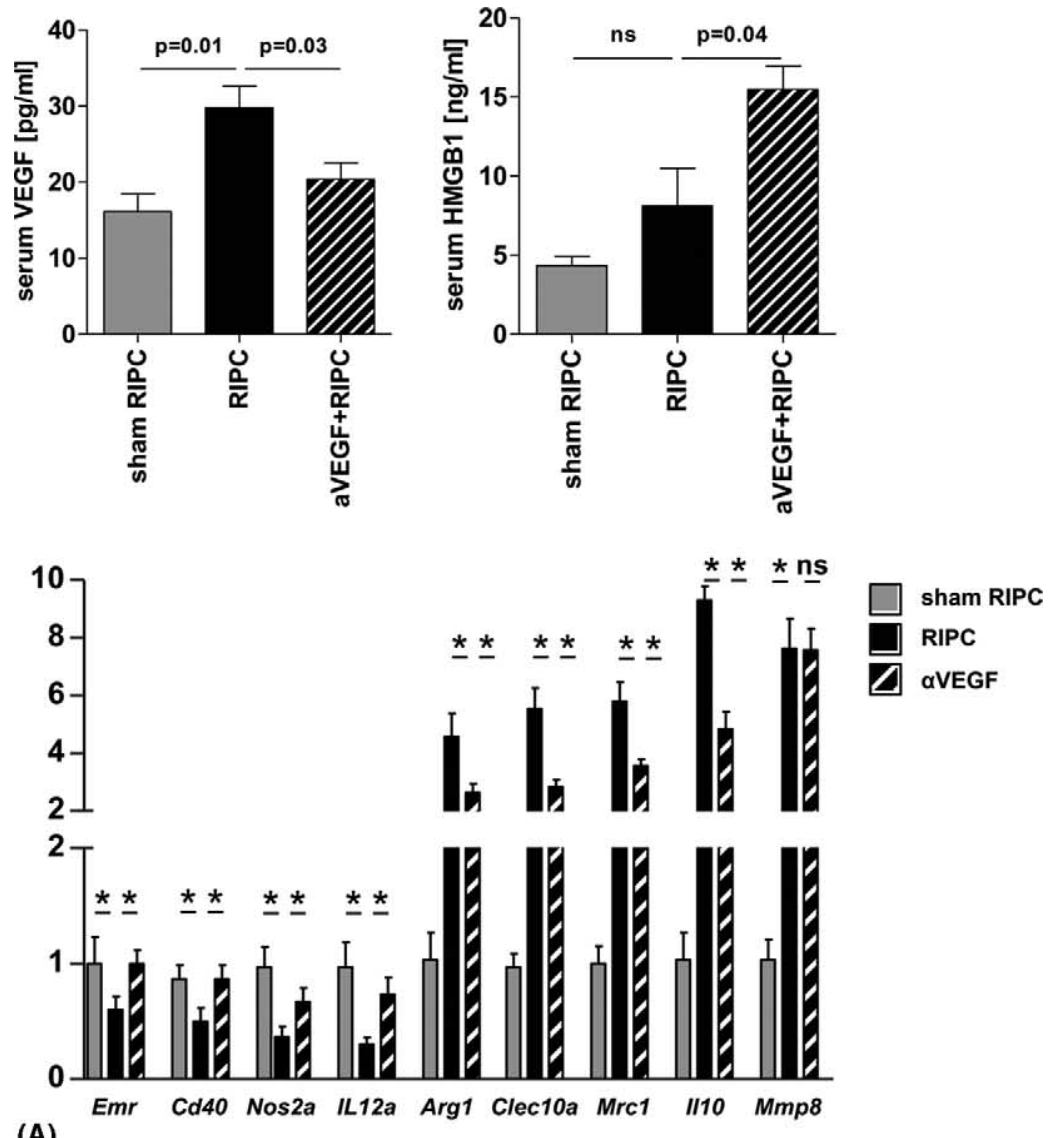

(A)

FIGURE 4. Effects of $\alpha$ Vegf pretreatment on RIPCinduced molecular changes and protection. A, Immediate effects on circulating Vegf, Hmgb1 (upper panels), macrophage polarization markers, and cytoprotective molecules (lower panel) following RIPC. B, Effects on liver injury parameters at $6 \mathrm{~h}$ after reperfusion subsequent to RIPC. ${ }^{*} P<0.05$. ns indicates nonsignificant.
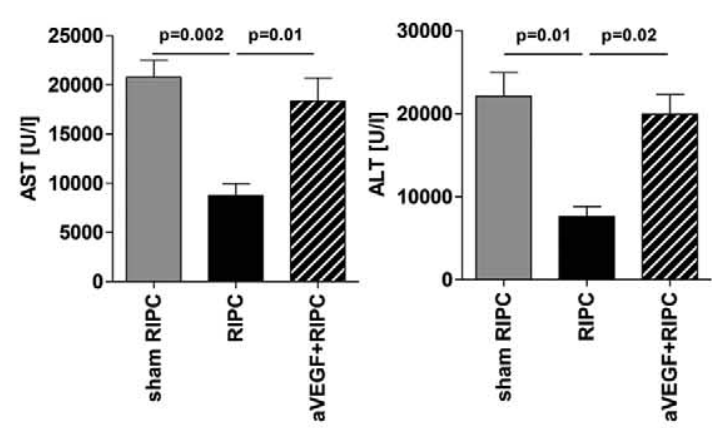

(B)
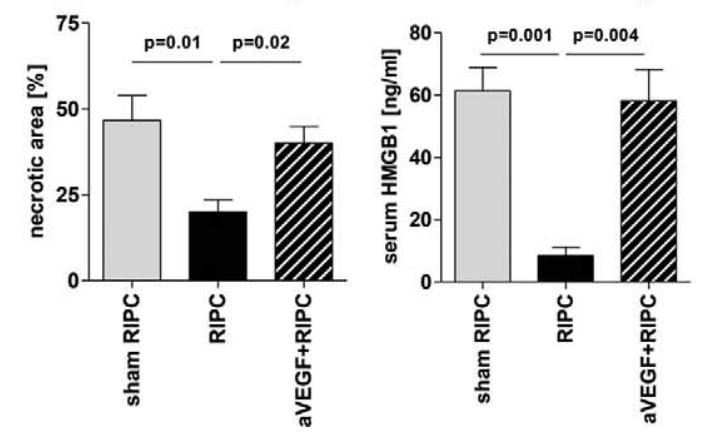

opioids) seem to interfere with the effects of preconditioning, either by counteracting or mimicking protective mechanisms. ${ }^{31-33}$ Two major trials on RIPC for cardiac surgery surprisingly concluded with the absence of benefits for patients. ${ }^{34,35}$ Although many reasons may come into play, a plausible explanation for failed protection through RIPC is that the pharmacological agents used in these 2 trials have neutralized any RIPC benefits or have pre-engaged protective routes otherwise activated through RIPC. ${ }^{33}$ Therefore, knowledge of the 

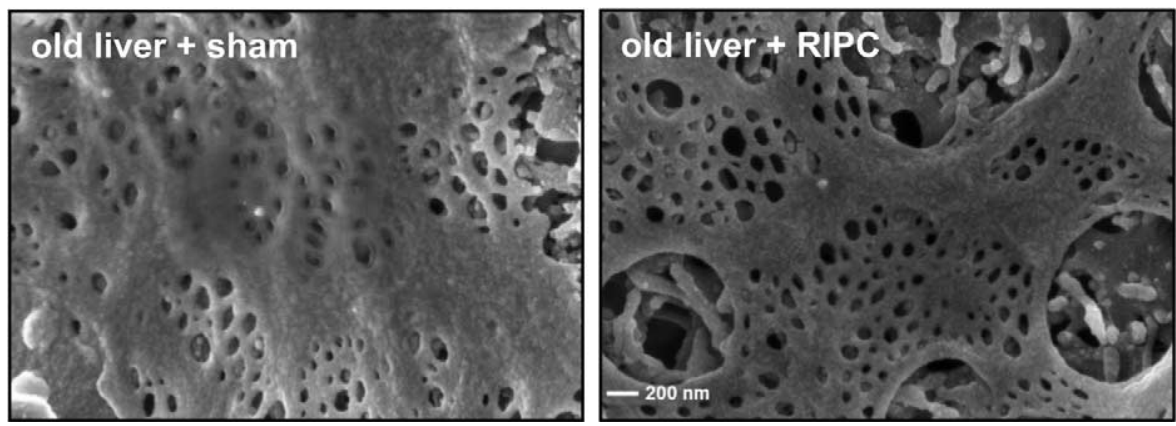

FIGURE 5. Effects of RIPC on sinusoidal endothelium. Note the regular and open fenestration patterns in sinusoids of RIPCtreated animals.

molecular paths that transmit and implement the many effects of RIPC is needed to define the clinical circumstances amenable to RIPC-conferred protection. In this regard, the serotonin-Vegf axis, clearly a key pathway of RIPC, may provide molecular markers (eg. circulating Vegf) to estimate whether, for example, specific anesthetics are suitable for a combination with RIPC.

Surgery on aged liver may be associated with additional factors limiting the application of RIPC. The incidence of chronic liver diseases such as fibrosis or hepatitis increases with age, and how these affect the efficacy of RIPC is largely unknown. For example, the increased angiogenic activity ongoing with liver fibrosis may interfere with Vegf-dependent protection induced by RIPC ${ }^{36}$; liver tumors, prime indications for surgery, on their own can produce significant amounts of Vegf and could mask beneficial RIPC effects. Indeed, direct Vegf injection may be used instead of RIPC, as we demonstrated for young mice. ${ }^{18}$ However, RIPC-induced Vegf elevations are likely more subtle and short-lived than a direct injection and hence may bear a smaller risk with regard to promotion of tumor-associated vasculature. Moreover, Vegf injection would not activate Vegf-independent pathways that may contribute to protection. These and related issues need to be experimentally addressed as to identify conditions wherein RIPC, or Vegf as a substitute, can unfold its powers. On the basis of our preclinical findings, we have initiated a clinical trial evaluating the effects of RIPC on morbidity and mortality post gastrointestinal and liver surgery (NCT02375269). Subanalyses should reveal which pathologies appear compatible with RIPC and whether RIPC indeed is protective also in the elderly.

In conclusion, our findings demonstrate the preclinical efficacy of RIPC in protecting old liver against IR. The effects RIPC imparts on old liver are chiefly mediated by circulating Vegf, resulting in the upregulation of cytoprotective molecules in liver, the promotion of an anti-inflammatory Kupffer cell phenotype, and an improved sinusoidal differentiation. The ease of its application in the clinic, the sparing of the target organ, and the large variety of potential beneficial effects render RIPC a highly attractive strategy for the reduction of postoperative complications. Particularly in the light of future demographics, our results advocate for a rapid evaluation of RIPC in liver surgery.

\section{ACKNOWLEDGMENT}

We would like to thank Jae-Hwi Jang, Udo Ungethüm, Eleonora Maurizio, and Pia Fuchs for excellent technical assistance.

\section{REFERENCES}

1. Selzner N, Rudiger H, Graf R, et al. Protective strategies against ischemic injury of the liver. Gastroenterology. 2003;125:917-936.

2. Zhai Y, Busuttil RW, Kupiec-Weglinski JW. Liver ischemia and reperfusion injury: new insights into mechanisms of innate-adaptive immune-mediated tissue inflammation. Am J Transplant. 2011;11:1563-1569.
3. Clavien PA, Petrowsky H, DeOliveira ML, et al. Strategies for safer liver surgery and partial liver transplantation. N Engl J Med. 2007;356:1545-1559.

4. Eltzschig HK, Eckle T. Ischemia and reperfusion-from mechanism to translation. Nat Med. 2011;17:1391-1401.

5. Zager RA, Baltes LA, Sharma HM, et al. Responses of the ischemic acute renal failure kidney to additional ischemic events. Kidney Int. 1984;26:689700 .

6. Murry CE, Jennings RB, Reimer KA. Preconditioning with ischemia: a delay of lethal cell injury in ischemic myocardium. Circulation. 1986;74:1124-1136.

7. Zhai Y, Petrowsky H, Hong JC, et al. Ischaemia-reperfusion injury in liver transplantation-from bench to bedside. Nat Rev Gastroenterol Hepatol. 2013;10:79-89.

8. Petrowsky H, McCormack L, Trujillo M, et al. A prospective, randomized, controlled trial comparing intermittent portal triad clamping versus ischemic preconditioning with continuous clamping for major liver resection. Ann Surg. 2006;244:921-928.

9. Selzner M, Selzner N, Jochum W, et al. Increased ischemic injury in old mouse liver: an ATP-dependent mechanism. Liver Transpl. 2007;13:382-390.

10. Furrer K, Rickenbacher A, Tian Y, et al. Serotonin reverts age-related capillarization and failure of regeneration in the liver through a VEGFdependent pathway. Proc Natl Acad Sci U S A. 2011;108:2945-2950.

11. Kulik U, Framke T, Grosshennig A, et al. Liver resection of colorectal liver metastases in elderly patients. World J Surg. 2011;35:2063-2072.

12. Shirabe K, Kajiyama K, Harimoto N, et al. Early outcome following hepatic resection in patients older than 80 years of age. World J Surg. 2009;33:19271932.

13. Rudiger HA, Kang KJ, Sindram D, et al. Comparison of ischemic preconditioning and intermittent and continuous inflow occlusion in the murine liver. Ann Surg. 2002;235:400-407.

14. Kang KJ, Jang JH, Lim TJ, et al. Optimal cycle of intermittent portal triad clamping during liver resection in the murine liver. Liver Transpl. 2004;10:794-801.

15. Clavien PA, Selzner M, Rudiger HA, et al. A prospective randomized study in 100 consecutive patients undergoing major liver resection with versus without ischemic preconditioning. Ann Surg. 2003;238:843-850.

16. O'Neill S, Leuschner S, McNally SJ, et al. Meta-analysis of ischaemic preconditioning for liver resections. Br J Surg. 2013;100:1689-1700.

17. Gill R, Kuriakose R, Gertz ZM, et al. Remote ischemic preconditioning for myocardial protection: update on mechanisms and clinical relevance. Mol Cell Biochem. 2015;402:41-49.

18. Oberkofler CE, Limani P, Jang JH, et al. Systemic protection through remote ischemic preconditioning is spread by platelet-dependent signaling in mice. Hepatology. 2014;60:1409-1417.

19. Jang JH, Kang KJ, Kang Y, et al. Ischemic preconditioning and intermittent clamping confer protection against ischemic injury in the cirrhotic mouse liver. Liver Transpl. 2008;14:980-988.

20. Rickenbacher A, Jang JH, Limani P, et al. Fasting protects liver from ischemic injury through Sirt1-mediated downregulation of circulating HMGB1 in mice. J Hepatol. 2014;61:301-308.

21. Raptis DA, Limani P, Jang JH, et al. GPR120 on Kupffer cells mediates hepatoprotective effects of omega3-fatty acids. J Hepatol. 2014;60:625-632.

22. Sorensen KK, Simon-Santamaria J, McCuskey RS, et al. Liver sinusoidal endothelial cells. Compr Physiol. 2015;5:1751-1774.

23. Xie G, Wang X, Wang L, et al. Role of differentiation of liver sinusoidal endothelial cells in progression and regression of hepatic fibrosis in rats. Gastroenterology. 2012;142:918-927. 
24. Ding BS, Nolan DJ, Butler JM, et al. Inductive angiocrine signals from sinusoidal endothelium are required for liver regeneration. Nature. 2010;468:310-315.

25. Totzeck M, Hendgen-Cotta U, Rassaf T. Concepts of hypoxic NO signaling in remote ischemic preconditioning. World J Cardiol. 2015;7:645-651.

26. Haapanen H, Herajarvi J, Arvola O, et al. Remote ischemic preconditioning protects the spinal cord against ischemic insult: an experimental study in a porcine model. J Thorac Cardiovasc Surg. 2015;S0022-5223:1253-1262.

27. Catma MF, Sesen H, Aydin A, et al. Remote ischemic preconditioning enhances fracture healing. J Orthop. 2015;12:168-173.

28. Gassman AA, Lewis MS, Bradley JP, et al. Remote ischemic preconditioning improves the viability of donor lipoaspirate during murine fat transfer. Plast Reconstr Surg. 2015;136:495-502.

29. Berger MM, Kohne H, Hotz L, et al. Remote ischemic preconditioning delays the onset of acute mountain sickness in normobaric hypoxia. Physiol Rep. 2015;3:e12325.

30. Barbosa TC, Machado AC, Braz ID, et al. Remote ischemic preconditioning delays fatigue development during handgrip exercise. Scand J Med Sci Sports. 2015;25:356-364.

31. Schlapfer M, Piegeler T, Dull RO, et al. Propofol increases morbidity and mortality in a rat model of sepsis. Crit Care. 2015;19:45.

32. Beck-Schimmer B, Bonvini JM, Schadde E, et al. Conditioning with sevoflurane in liver transplantation: results of a multicenter randomized controlled trial. Transplantation. 2015;99:1606-1612.

33. Zaugg M, Lucchinetti E. Remote ischemic preconditioning in cardiac surgery-ineffective and risky? N Engl J Med. 2015;373:1470-1472.

34. Hausenloy DJ, Candilio L, Evans R, et al. Remote ischemic preconditioning and outcomes of cardiac surgery. N Engl J Med. 2015;373:1408-1417.

35. Meybohm P, Bein B, Brosteanu O, et al. A multicenter trial of remote ischemic preconditioning for heart surgery. N Engl J Med. 2015;373:1397-1407.

36. Zhang Z, Zhang F, Lu Y, et al. Update on implications and mechanisms of angiogenesis in liver fibrosis. Hepatol Res. 2015;45:162-178.

\section{DISCUSSANTS}

\section{Rogiers (Gent, Belgium):}

I would like to congratulate you as well as your co-investigators on this fine piece of experimental work. In this work, you demonstrate the preclinical efficacy of remote ischemic preconditioning (RIPC) in protecting old livers against ischemia reperfusion injury (IR) in a mice model.

My first question relates to age. Is there strong evidence that age-dependent problems with recovery from IR are also present in humans and what is the critical age? Your mice are 20 to 24 months of age. What age in humans would that relate to?

Damage by chemotherapy may be more relevant than age for the liver. Can you speculate if this works in chemotherapydamaged livers?

My other question is whether it is the age of the liver or the age of the patient, which is relevant. This might have importance in the transplantation setting where the median donor age is steadily increasing and may impact on graft quality. Could you speculate on the importance of your work for the transplantation patients?

\section{Response From P. Limani (Zurich, Switzerland):}

Thank you for your kind comments and relevant questions.

There is good evidence in humans for ischemic injury increasing with age [Transplant Proc 1995;27:1181-3. and Dig Dis Sci 2016;61:626-35]. We further showed that ischemic preconditioning loses its effects in patients above 65 years, suggesting this is a critical age (Ann Surg 2006;244:921-8).

As for mouse age, 20 months correspond to about 80 years in humans.
How RIPC would affect injury levels in chemotherapy-damaged livers is difficult to predict. Oxaliplatin-based regimens often infer sinusoidal injury. In our study, RIPC improved sinusoidal integrity, it hence is tempting to speculate that RIPC might be of benefit here. Our preliminary findings further indicate RIPC does improve the regenerative capacity of liver, perhaps aiding the recovery of chemically damaged parenchyme as well.

Finally, while RIPC and Vegf may also affect the patient body, we observed a direct, protection-associated effect on age-related pseudocapillarization in liver. As we have previously shown, Vegf induction and reversal of pseudocapillarization also improve the regenerative capacity of aged liver [PNAS USA 2011;108:2945-50]. These hepatic effects should be beneficial for the transplantation of old grafts obtained from old donors.

\section{A. Pinna (Bologna, Italy):}

Have you given a thought to infused plasma from remote ischemia preconditioning animals to control animals?

What do you think about infusing ischemic protective cytokines in IR animals to see if there is a difference in the damage?

\section{Response From P. Limani (Zurich, Switzerland):}

Thank you for this question. Indeed, we have not studied RIPC plasma, but this is an interesting thought. We would expect that RIPC plasma is likewise protective. Whereas circulating Vegf clearly is crucial for protection, it is likely other factors contribute. This could be researched with plasma experiments. For example, if whole plasma is more protective, additional protective molecules such as cytokines should be present. Likewise, the latter would be the case if RIPC plasma from animals pretreated with an anti-Vegf antibody still confers some protection. Such plasma then could be systematically screened for candidate molecules such as cytokines.

\section{T. van Gulik (Amsterdam, The Netherlands):}

This RIPC strategy is a fascinating phenomenon. You very nicely demonstrated morphologically with scanning electron microscopy the fenestrations of the sinuosoidal endothelial cells showing defects as a result of postischemic reperfusion. We know that in particular it is the space of Disse behind the endothelial cells that is injured. Did you look with transmission electron microscopy at the sinusoidal endothelial cell lining to observe these spaces of Disse behind the endothelial cells that is injured?

Your hypothesis is that you improved perfusion of the liver by RIPC. Did this translate into decreased portal pressure? The endpoint is portal pressure which will increase when there is endothelial injury and which will decrease if you improve microcirculation of the liver?

\section{Response From P. Limani (Zurich, Switzerland):}

Thank you for your important comments and questions. We did not examine the space of Disse, because our study focused on pseudocapillarization of the sinusoids. We hence examined liver only by scanning EM. Clearly, the space of Disse, likely also affected by aging, should be considered in future studies on RIPC and old liver.

RIPC is known to improve hepatic microcirculation, and dilated fenestration has been associated with reduced portal pressure. We therefore expect that RIPC, opening fenestrae, will also reduce portal pressure. We have not investigated this further, but we agree that portal assessment would be important in establishing the above associations. 